Disclosure of Interests: None declared

DOI: 10.1136/annrheumdis-2019-eular.6778

\section{AB0589 ANCA ASSOCIATED VASCULITIS: OUR EXPERIENCE FROM A TERITIARY CARE CENTER OVER 10 YEARS}

Anjani Gummadi ${ }^{1}$, Ankita Singh ${ }^{1}$, Sandesh Guleria ${ }^{2}$, Pandiarajan Vignesh ${ }^{2}$, Anju Gupta ${ }^{1}$, Deepti Suri ${ }^{2}$, Ankur Jindal ${ }^{3}$, Biman Saikia ${ }^{4}$, Amit Rawat ${ }^{3}$, Surjit Singh ${ }^{2} .{ }^{1}$ Post Graduate Institute of Medical Education and Research, Chandigarh, Pediatrics, Advanced Pediatric centre, Chandigarh, India; ${ }^{1}$ Post Graduate Institute of Medical Education and Research, Chandigarh, Pediatrics, Advanced Pediatric centre, Chandigarh, India; ${ }^{3}$ Post Graduate Institute of Medical Education and Research, Chandigarh, Chandigarh, India; ${ }^{4}$ Post Graduate Institute of Medical Education and Research, Chandigarh, Department of Immunopathology, Chandigarh, India

Background: The anti-neutrophil cytoplasmic antibody (ANCA)- associated vasculitides (AAV) are a group of disorders characterized by necrotizing inflammation of the small to medium vessels in association with autoantibodies. Childhood ANCA vasculitides are rare but can cause organ or even life-threatening systemic vasculitis. Children most frequently present with rapidly evolving, severe disease.

Objectives: To describe the clinical spectrum of ANCA associated vasculitis, the treatment given and follow up

Methods: A single-centre retrospective analysis of ANCA associated vasculitis over a period of 10 years from 2008 to 2018

Results: Six children (2 boys; 4 girls) were diagnosed to have AAV during this period. Median age at diagnosis was 11.25 years (range 8-18 years). Median delay in diagnosis was 1.5 months (range 1-8 months). Presenting clinical features included lung disease- 4 children; arthritis- 3 (knee, right ankle-left $3^{\text {rd }} \mathrm{MCP}$, left elbow-knee) hemoptysis- 2 children; ear discharge- 1; redness of eye-1; oliguria, gross hematuria-2; anasarca2; 2 children had concomitant neurological symptoms (left foot drop, quadriparesis with seizures). All children had fever at presentation. Laboratory investigations showed elevated erthrocyte sedimentation rate(ESR) and C-reactive protein(CRP). ANCA testing was positive in 6 children (3 c-ANCA, +PR3, 3 p-ANCA, +MPO). Deranged renal function tests, proteinuria, microscopic hematuria) were seen in 4 out of 6 children. Renal biopsy showed pauci immune glomerulonephritis in 4 cases who presented with nephritis. All patients were treated with 5 pulses of intravenous methylprednisolone $(30 \mathrm{mg} / \mathrm{kg} /$ day $)$ with tapering doses of oral steroids. Intravenous cyclophosphamide pulses was given in 5 children while 1 received additional plasmapheresis (PLEX)- 8 cycles. Mintainence therapy included azathioprine(given in 5 children), low-dose prednisolone. Thrombocytopenia was noted in 1 child while on azathioprine; this was subsequently substituted with mycophenolate mofetil. Two children have been lost to follow-up. Median duration of follow-up is 35.5 months (range 8-124 months). All 4 children have attained remission with good compliance and there are no relapses.

Conclusion: While an evidence base treatment of AAV in children is limited, our data suggests that prolonged remission can be achieved with aggressive immnosupressive therapy, however prognosis of childhood AAV remains guarded.

Table: Characteristics of children enrolled in study

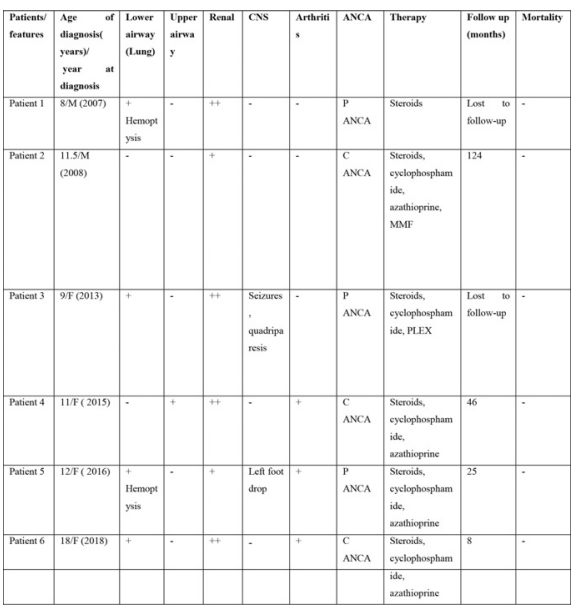

Disclosure of Interests: None declared DOI: 10.1136/annrheumdis-2019-eular.8244

\section{$\mathrm{AB} 0590$ \\ A SINGLE CENTER RETROSPECTIVE ANALYSIS OF EFFICACY AND SAFETY BETWEEN LOW-DOSE VERSUS HIGH-DOSE RITUXIMAB AS REMISSION INDUCTION THERAPY IN JAPANESE PATIENTS WITH ANCA-ASSOCIATED VASCULITIS}

Kei Hirose, Manami Hirata, Yuriko Yamamura, Akiko Ueno, Masahiro Yamamura. Okayama Saiseikai General Hospital, Center for Rheumatology, Okayama city, Japan

Background: Administration of four once-weekly doses of $375 \mathrm{mg} / \mathrm{m}^{2}$ rituximab (RTX) has been indicated for ANCA-associated vasculitis (AAV) as remission induction therapy. However, randomized controlled trial for Japanese AAV patients have never been conducted, although Japanese AAV patients are characterized by the predominance of elderly patients with microscopic polyangiitis (MPA)

Objectives: To compare the efficacy and safety between low-dose versus high-dose RTX therapy as remission induction therapy in Japanese patients with AAV.

Methods: A single center retrospective analysis of 27 consecutive AAV patients with RTX therapy was performed. Clinical and laboratory variables at diagnosis, rates of complete remission (CR), defined as Birming ham Vasculitis Activity Score (BVAS) $=0$ and prednisone $<7.5 \mathrm{mg} /$ day, adverse effects, and vasculitis relapses following RTX use.

Results: Twenty-five MPA patients and 2 GPA patients $(14$ males and 13 females) were included in the present study. Twenty-six patients were positive for MPO-ANCA. Their median age was 77 years (range: 40-85 years). Treatments were determined according to the discretion of the attending physician. As remission induction therapy, 18 patients were treated with once or twice (1/2) RTX infusions $\left(375 \mathrm{mg} / \mathrm{m}^{2}\right)$, while 9 patients with 3 or 4 times (3/4) RTX infusions. At 6 months, $55.6 \%$ of the $1 / 2$ infusion group (10/18) and $44.4 \%$ of the $3 / 4$ infusion group (4/9) reached CR. At 6 months, mean PSL levels were $7.8 \mathrm{mg} /$ day in the $1 / 2$ infusion group and $6.6 \mathrm{mg} /$ day in the $3 / 4$ infusion group. At 18 months, $88.9 \%$ of the $1 / 2$ infusion group $(16 / 18)$ and $77.8 \%$ of the $3 / 4$ infusion group (7/9) were survived. $0 \%$ of the $1 / 2$ infusion group (0/18) and $33.3 \%$ of the $3 / 4$ infusion group (3/9) were relapsed. Severe adverse effects occurred in $38.9 \%$ of the $1 / 2$ infusion group $(7 / 18)$ and in $22.2 \%$ of the $3 / 4$ infusion group (2/9).

Conclusion: Our retrospective analysis indicated that cumulative $\mathrm{CR}$ rates and PSL tapering did not significantly differ between low-dose versus high-dose RTX as remission induction therapy in Japanese AAV patients, mostly elderly MPA patients, although there was no significant difference in severe adverse effects such as opportunistic infections between them. Disclosure of Interests: None declared DOI: 10.1136/annrheumdis-2019-eular.4450

\section{AB0591 POSITIVE PREDICTIVE VALUE OF THE GIANT CELL ARTERITIS DIAGNOSIS IN THE DANISH NATIONAL PATIENT REGISTRY: A VALIDATION STUDY}

Peter Hiort ${ }^{1}$, Philip Therkildsen ${ }^{1}$, Berit Dalsgaard Nielsen ${ }^{1}$, Ib Hansen $^{1}$, Annette de Thurah ${ }^{2}$, Ellen-Margrethe Hauge ${ }^{1} .{ }^{1}$ Aarhus University Hospital, Rheumatology, Aarhus, Denmark, ${ }^{2}$ Aarhus University Hospital, Aarhus, Denmark

Background: Giant cell arteritis (GCA) is the most frequent systemic vasculitis[1]. The diagnosis is clinical and based on symptoms, histopathology, biochemistry and imaging. In Denmark, diagnostic codes for all inand out-patient hospital diagnoses are registered in the Danish National Patient Registry (DNPR) [2]. Since GCA can be difficult to diagnose and treatment is initiated on suspicion, we hypothesized that the overall positive predictive value (PPV) of the GCA diagnosis code in the DNPR is low. High data quality is important for future epidemiological research in GCA.

Objectives: To establish PPV of the diagnostic code of GCA in the DNPR. Furthermore, to identify characteristics associated with a high PPV of the diagnostic code.

Methods: 293 patients aged $\geq 50$ years with a first-time register-based GCA diagnosis were included from the DNPR in the period January 2012-January 2018. Patients were sampled based on the ICD-10 codes (M31.5 and M31.6) from two regional hospitals and one university hospital in the Central Region of Denmark. As gold standard we used the medical records (including biochemistry, histopathology and imaging results) and categorized each patient as true GCA or non-GCA. Based on the data from the prescription database, patients were divided into four categories depending on the number of prednisolone prescriptions they received. Two independent investigators (PH and PT) reviewed the medical records. In case of disagreement the final diagnosis was reached by consensus or by expert opinion (ITH). To test how the PPV varied, 